\title{
About a certain relation between two polynomials of the same degree
}

\author{
TADEUSZ PIWOWARCZYK
}

\begin{abstract}
This article presents several different methods for solving the problem of how to find a certain relation defined in chapter 2 . The first method deals with the identities known in the theory of symmetric polynomials as the elements of a certain vector space. The second method is designed around the matrix transformations between symmetric polynomials. The third method is designed around the property of a linear operator and its characteristic polynomial. The fourth method is designed in the area of complex numbers, and introduces the multiplication group of 'complex roots of one'. Significant improvement in the third and fourth method is made by introducing so called 'block method'. It facilitates all calculations by making them much shorter. The article ends with an example showing symmetry and regularity of all procedures. Finally, the article shows how to solve the problem for any degree $n$ of the polynomial, and for any degree $k$. At the end of the paper solutions for $n \leqslant 5$ and $k \leqslant 5$ are tabulated.
\end{abstract}

Key words: roots of polynomials, symmetric polynomials, companion matrix, eigenvalues, transformations

\section{Introduction}

The relation $R_{k}$ defined below plays an important role in the analysis of the rational functions (transmittances). Chronologically speaking, this problem was taken into consideration in 1958, and then the attempts to solve were published in [8]. One can find there the Encke roots and the Graef-Łobaczewski method. By means of fast convergent procedures we can estimate not only the sign of the roots, but also the numerical values of the roots (stability, simplification of the rational functions and other applications). It should be noticed at this point that the simplification of the rational function can also be solved by using other approaches (spectral notation of symmetric polynomials) [10]. Also the relation $R_{k}$ is used to analyze the polynomials with fractional powers.

The problem how to determine the relation $R_{k}$ will be solved in this article by using several methods. All methods are connected with the theory of polynomials of multiple

The Author is with Cracow Uniwersity of Technology, Faculty of Electrical and Computer Engineering, Kraków 31-155, Warszawska 24. E-mail: ewa.lechpi@op.pl

Author would like to express the gratitude to Professor Henryk Górecki for his inspiration, guidance and assistance which made writing of this paper possible.

Received 26.01.2011. Revised 14.03.2001. 
variables. The procedures proposed in the article are ready to be applied for $n \leqslant 5$ and $k \leqslant 5$. For higher degrees, especially the degree $k$, the necessary transformations are time consuming so the application of the computer calculations are necessary. Tab. 3 at the end of the paper shows the solutions up to 5-th degree. In other words, Tab. 3. presents the coefficients of the second polynomial as functions the coefficients of the first one. All the functions are computed and checked by using the methods introduced in this paper.

Of all the formulas presented in this paper only two can be found in the literature of the subject. The first one comes from [1] where its author, J. Ambrosiewicz, analyzes the natural powers of the companion matrices $C$ raised to a natural power $n$. They are connected with the power sums of eigenvalues of a given linear operator (the trace of the companion matrix in the natural power $\operatorname{tr} C^{n}$ ). In the spectral notation we have here the formula (20). The second formula which can be found in literature, for example in [6], is the matrix formula (26) written in the present paper in the spectral notation. The other formulas, definitions and theorems, which occur in this paper are the author's own original and genuine contributions to the subject.

\section{Definition of the relation $R_{k}$}

Two polynomials of the same degree $n$

$$
\begin{array}{ll}
f(s)=a_{0} \cdot s^{n}+a_{1} \cdot s^{n-1}+\ldots+a_{n-1} \cdot s+a_{n}, & a_{i} \in \mathfrak{R} \\
g(s)=b_{0} \cdot s^{n}+b_{1} \cdot s^{n-1}+\ldots+b_{n-1} \cdot s+b_{n}, & b_{i} \in \mathfrak{R}
\end{array}
$$

satisfy the relation $R_{k}$ if two equations $f(s)=0$ and $g(s)=0$ have the roots respectively given in the following sets:

$$
\begin{aligned}
& \left\{s_{1}, s_{2}, \ldots, s_{n}\right\}, \\
& \left\{s_{1}^{k}, s_{2}^{k}, \ldots, s_{n}^{k}\right\} .
\end{aligned}
$$

To determine the relation $R_{k}$ means finding the following $n$ functions

$$
b_{i}\left(a_{0}, a_{1}, \ldots, a_{n}\right), \quad i=1,2, \ldots, n .
$$

The coefficients of (1) in the theory of symmetric polynomials in a spectral notation (see Appendix) [11],[12],[13] take the form

$$
a_{i}=(-1)^{i-1} \cdot((\underbrace{1,1, \ldots, 1}_{i}))
$$

and the coefficients of (2) take the form

$$
b_{i}=(-1)^{i-1} \cdot((\underbrace{k, k, \ldots, k}_{i})) \text {. }
$$


This fact seems to be obvious. The expressions (5) depend of following identities

$$
((\underbrace{k, k, \ldots, k}_{i}))=f_{i, k}[((1)),((1,1)), \ldots,((\underbrace{11, \ldots, 1}_{i \cdot k}))] .
$$

Table 4. Identities of the type (8).

\begin{tabular}{|l|l|l|r|}
\hline$((2))$ & $=$ & $-2((1,1))+((1))^{2}$ & $(9)$ \\
\hline$((2,2))$ & $=$ & $2((1,1,1,1))-2((1,1,1))((1))+((1,1))^{2}$ & $(10)$ \\
\hline$((2,2,2))$ & $=$ & $-2((1,1,1,1,1,1))+2((1,1,1,1,1))((1))-2((1,1,1,1))((1,1))+((1,1,1))^{2}$ & $(11)$ \\
\hline$((3))$ & $=$ & $3((1,1,1))-3((1,1))((1))+((1))^{3}$ & $(12)$ \\
\hline$((3,3))$ & $=$ & $3((1,1,1,1,1,1))-3((1,1,1,1,1))((1))-3((1,1,1,1))((1,1))+$ & $(13)$ \\
& & $3((1,1,1,1))((1))^{2}+3((1,1,1))^{2}-3((1,1,1))((1,1))((1))+((1,1))^{3}$ & \\
\hline$((4))$ & $=$ & $-4((1,1,1,1))+4((1,1,1))((1))+2((1,1))^{2}-4((1,1))((1))^{2}+((1))^{4}$ & $(14)$ \\
\hline$((5))$ & $=$ & $5((1,1,1,1,1))-5((1,1,1,1))((1))-5((1,1,1))((1,1))+5((1,1,1))((1))^{2}+$ & $(15)$ \\
& & $5((1,1)) 2((1))-5\left((1,1)((1))^{3}+((1))^{5}\right.$ & \\
\hline$((6))$ & $=$ & $-6((1,1,1,1,1,1))+6((1,1,1,1,1))((1))+6((1,1,1,1))((1,1))-$ & $(16)$ \\
& & $6((1,1,1,1))((1))^{2}+3((1,1,1))^{2}-12((1,1,1))((1,1))((1))+$ & \\
$6((1,1,1))((1))^{3}-2((1,1))^{3}+9((1,1))^{2}((1)) 2-6((1,1))((1))^{4}+((1))^{6}$ & \\
\hline
\end{tabular}

\section{The first method}

In the monograph [11] we can find some identities of the type (8). They are displayed in Tab. 1.

Using the identities presented in Tab. 1 i.e. (9),(10),(11),(12) and (13) we can solve three cases only:

$$
\begin{gathered}
s^{2}+a_{1} \cdot s+a_{2}, \quad k=2, \\
s^{2}+a_{1} \cdot s+a_{2}, \quad k=3, \\
s^{3}+a_{1} \cdot s^{2}+a_{2} \cdot s+a_{3}, \quad k=2 .
\end{gathered}
$$

To solve the problem for any $n$ and any $k$ we should know firstly how to extend the Tab. 1. This can be done using different methods. In [11] a vector space is defined which elements are symmetric polynomials. Among them there are identities (8). However, in order to find such identities for a higher degree we need to apply time consuming procedures. An algebra generalization of this method is presented below as the second method. 


\section{The second method}

In the monograph [11] one can find a matrix procedure which shows how to calculate the polynomial $((k))$ if the polynomials $((1)),((1,1)), \ldots,((\underbrace{11, \ldots, 1}_{k}))$ are given.

$$
((k))=
$$

$$
\operatorname{tr}\left[\begin{array}{cccccc}
0 & 1 & 0 & \cdot & 0 & 0 \\
0 & 0 & 1 & \cdot & 0 & 0 \\
0 & 0 & 0 & \cdot & 0 & 0 \\
0 & 0 & 0 & \cdot & 0 & 0 \\
\left.\cdot(-1)^{k}(\underbrace{1,1, \ldots, 1}_{k})\right) & \left.(-1)^{k-1}(\underbrace{1,1, \ldots, 1}_{k-1})\right) & \left.(-1)^{k-2}(\underbrace{(1,1, \ldots, 1}_{k-2})\right) & \cdot & \cdot((1,1)) & -((1))
\end{array}\right]^{k}
$$

Symbol $t r$ stands for the trace of a matrix. The procedure (20) can be performed by another matrix formula

$$
\begin{aligned}
& {\left[\begin{array}{c}
T_{1} \\
T_{2} \\
T_{3} \\
T_{4} \\
\cdot \\
T_{k}
\end{array}\right]=\left[\begin{array}{cccccc}
1 & 0 & 0 & 0 & \cdot & 0 \\
-V_{1} & 1 & 0 & 0 & \cdot & 0 \\
V_{2} & -V_{1} & 1 & 0 & \cdot & 0 \\
-V_{3} & V_{2} & -V_{1} & 1 & \cdot & 0 \\
\cdot & \cdot & \cdot & \cdot & \cdot & \cdot \\
(-1)^{k} \cdot V_{k} & (-1)^{k-1} \cdot V_{k-1} & (-1)^{k-2} \cdot V_{k-2} & (-1)^{k-4} \cdot V_{k-3} & \cdot & 1
\end{array}\right]^{-1}} \\
& {\left[\begin{array}{c}
V_{1} \\
-2 \cdot V_{2} \\
3 \cdot V_{3} \\
-4 \cdot V_{4} \\
\cdot \\
(-1)^{k+1} \cdot k \cdot V_{k}
\end{array}\right]}
\end{aligned}
$$

where

$$
\begin{gathered}
V_{k}=((\underbrace{1,1, \ldots, 1}_{k}), \\
T_{k}=((k)) .
\end{gathered}
$$

Matrix formula (21) was designed especially for the purpose of this paper. It simplifies determination of symmetric polynomials $T_{1}, T_{2}, \ldots, T_{k}$ i.e. $((1)),((2)), \ldots,((k))$. Formula (21) plays a supplementary role in this paper, and its inductive proof is omitted here. 
Once we know the functions

$$
((k))=G[((1)),((1,1)), \ldots,((\underbrace{11, \ldots, 1}_{k}))]
$$

we can compute the final function $((\underbrace{k, k, \ldots, k}_{i}))$. In the monograph [1] the following symmetric polynomial was analyzed

$$
\begin{aligned}
& ((\underbrace{k, k, \ldots, k}_{i}))=f_{k, n}[((k)),((2 \cdot k)), \ldots,((i \cdot k)], \\
& (\underbrace{k, k, \ldots, k}_{i})=\frac{1}{i !} \cdot\left|\left[\begin{array}{cccccc}
((k)) & ((2 \cdot k)) & ((3 \cdot k)) & ((4 \cdot k)) & \cdot & ((i \cdot k)) \\
1 & ((k)) & ((2 \cdot k)) & ((3 \cdot k)) & \cdot & (((i-1) \cdot k)) \\
0 & 2 & ((k)) & ((2 \cdot k)) & \cdot & (((i-2) \cdot k)) \\
0 & 0 & 3 & ((k)) & \cdot & (((i-3) \cdot k)) \\
\cdot & . & . & . & . & \cdot \\
0 & 0 & 0 & 0 & . & ((k))
\end{array}\right]\right| .
\end{aligned}
$$

Using the matrix formula (26) we will be able to determine all the formulas which will appear in the Tab. 3 at the end of the paper. However, the procedures are still time consuming and tedious.

\section{The third method}

The method presented below is called a 'linear operator approach'. Let define the companion matrix $C$ and the identity matrix $I$.

$C=\left[\begin{array}{cccccc}0 & 1 & 0 & 0 & . & 0 \\ 0 & 0 & 1 & 0 & . & 0 \\ 0 & 0 & 0 & 1 & . & 0 \\ 0 & 0 & 0 & 0 & . & 0 \\ \cdot & . & . & . & . & . \\ -a_{n} & -a_{n-1} & -a_{n-2} & -a_{n-3} & . & -a_{1}\end{array}\right], I=\left[\begin{array}{cccccc}1 & 0 & 0 & 0 & . & 0 \\ 0 & 1 & 0 & 0 & . & 0 \\ 0 & 0 & 1 & 0 & . & 0 \\ 0 & 0 & 0 & 1 & . & 0 \\ . & . & . & . & . & . \\ 0 & 0 & 0 & 0 & . & 1\end{array}\right]$.

For given polynomials (1) and (2) we can write them as the characteristic polynomials

$$
f(s)=|C-I \cdot s|
$$

and consequently,

$$
g(s)=\left|C^{k}-I \cdot s\right| .
$$


Formula (29) is obvious. Detailed analysis of (29) can be found in [11] and [1]. Using (29) we can determine all the formulas which will be shown in the Tab. 3 at the end of this paper. If applied for the higher degrees of the function this approach is still very tedious.

\section{The fourth method}

The method described in section 5, the 'linear operator method' is effective and clear from algebra point of view, but for greater $n$, and especially for larger $k$, it is much time consuming and tedious. Simplification is then necessary. For this purpose we should consider modification of the formula (29) as follows

$$
g\left(s^{k}\right)=\left|C^{k}-I \cdot s^{k}\right| .
$$

Thus, properly speaking, we analyze the power $k$ of a given linear operator. To analyze (30) we introduce the 'roots of one' in a complex number. For a natural number $k$ define $K$ as

$$
K=\cos \frac{2 \cdot \pi}{k}+i \cdot \sin \frac{2 \cdot \pi}{k} .
$$

The complex value $K$ has the following property

$$
K+K^{2}+K^{3}+\ldots+K^{k}=0, \quad K^{k}=1 .
$$

In complex numbers the formula (30) can be factored

$$
g\left(s^{k}\right)=\left|C^{k}-I \cdot s^{k}\right|=\left|(C-I \cdot s \cdot K) \cdot\left(C-I \cdot s \cdot K^{2}\right) \cdot \ldots \cdot\left(C-I \cdot s \cdot K^{k}\right)\right|
$$

thus

$$
g\left(s^{k}\right)=\left|C^{k}-I \cdot s^{k}\right|=|C-I \cdot s| \cdot|C-I \cdot K \cdot s| \cdot \ldots \cdot\left|C-I \cdot s \cdot K^{k-1}\right|
$$

and finally

$$
g\left(s^{k}\right)=\left|C^{k}-I \cdot s^{k}\right|=f(s) \cdot f(K \cdot s) \cdot \ldots \cdot f\left(K^{k-1} \cdot s\right) .
$$

The points (33), (34) and (35) can be taken as steps leading to the proof of the basic formula (35). In this paper the formula (35) is called 'the complex number formula'. All the solutions presented in Tab. 3 are checked according this formula. In (35) $s^{k}$ should be replaced by $s$. This substitution is always possible. We can also demonstrate the formula (35) in some other way. After the right hand side of (35) is factored by introducing the appropriate roots of the given functions, one can write the following product: 


$$
\begin{gathered}
f(s \cdot)=\left(s-s_{1}\right) \cdot\left(s-s_{2}\right) \cdot \ldots \cdot\left(s-s_{n}\right), \\
f(s \cdot K)=\left(s \cdot K-s_{1}\right) \cdot\left(s \cdot K-s_{2}\right) \cdot \ldots \cdot\left(s \cdot K-s_{n}\right), \\
\vdots \\
\left.f\left(s \cdot K^{k-1}\right)=\left(s \cdot K^{k-1}-s_{1}\right) \cdot\left(s \cdot K^{k-1}-s_{2}\right) \cdot \ldots \cdot\left(s \cdot K^{k-1}-s_{n}\right)\right) .
\end{gathered}
$$

By changing the order in the above product we arrive at the complex numbers

$$
\begin{gathered}
\left(s-s_{1}\right) \cdot\left(s \cdot K-s_{1}\right) \cdot \ldots \cdot\left(s \cdot K^{k-1}-s_{1}\right)=s^{k}-s_{1}^{k}, \\
\left(s-s_{2}\right) \cdot\left(s \cdot K-s_{2}\right) \cdot \ldots \cdot\left(s \cdot K^{k-1}-s_{2}\right)=s^{k}-s_{2}^{k}, \\
\vdots \\
\left(s-s_{n}\right) \cdot\left(s \cdot K-s_{n}\right) \cdot \ldots \cdot\left(s \cdot K^{k-1}-s_{n}\right)=s^{k}-s_{n}^{k},
\end{gathered}
$$

thus

$$
\left(s^{k}-s_{1}^{k}\right) \cdot\left(s^{k}-s_{2}^{k}\right) \cdot \ldots \cdot\left(s^{k}-s_{n}^{k}\right)=g\left(s^{k}\right) .
$$

Expression (43) ends the demonstration of the formula (35) i.e.

$$
g\left(s^{k}\right)=f(s) \cdot f(K \cdot s) \cdot \ldots \cdot f\left(K^{k-1} \cdot s\right) .
$$

Formula (43) makes the procedure much shorter, and delivers a set of interesting properties of $g(s)$. Further simplifications are possible by introducing a subdivision of the given function $f(s)$ in $k$ blocks.

\section{Block method}

The method described below called the 'block method' can be applied to the 'matrix method, as well as to the 'complex number' method. We decompose the given function $f(s)$ of the degree $n$ into $k$ following blocks denoted by the functions $A_{1}(s), A_{2}(s), \ldots, A_{k}(s)$.

$$
\begin{gathered}
A_{1}(s)=s^{n}+a_{k} \cdot s^{n-k}+\ldots \\
A_{1}(s)=a_{1} \cdot s^{n-1}+a_{k+1} \cdot s^{n-k-1}+\ldots, \\
\vdots \\
A_{k}(s)=a_{k} \cdot s^{n-k}+a_{2 \cdot k} \cdot s^{n-2 \cdot k}+\ldots
\end{gathered}
$$

Obviously

$$
f(s)=A_{1}(s)+A_{2}(s)+\ldots+A_{k}(s)
$$


When analyzing (29), as well as (43) we get (48) and (49). By operating only with the block symbols $A_{1}, A_{2}, \ldots, A_{k}$ we get the formal function $F\left(A_{1}, A_{2}, \ldots, A_{k}\right)$. It is evident that the function (49) is equivalent to the notation (43). The below matrix formula is the consequence of (29) and (43):

$$
\begin{aligned}
& F\left(A_{1}, A_{2}, \ldots, A_{k}\right)= \\
& (-1)^{k} \cdot\left|\left[\begin{array}{cccccc}
0 & 1 & . & 0 & 0 & 0 \\
\cdot & \cdot & \cdot & . & \cdot & \cdot \\
0 & 0 & . & 1 & 0 & 0 \\
0 & 0 & . & 0 & 1 & 0 \\
0 & 0 & . & 0 & 0 & 1 \\
\left(-A_{k}+1\right) & -A_{k-1} & . & -A_{3} & -A_{2} & -A_{1}
\end{array}\right]^{k}-\left[\begin{array}{cccccc}
1 & 0 & . & 0 & 0 & 0 \\
\cdot & \cdot & \cdot & \cdot & \cdot & . \\
0 & 0 & . & 0 & 0 & 0 \\
0 & 0 & \cdot & 1 & 0 & 0 \\
0 & 0 & . & 0 & 1 & 0 \\
0 & 0 & . & 0 & 0 & 1
\end{array}\right]\right|
\end{aligned}
$$

In a complex number domain the block formula $F\left(A_{1}, A_{2}, \ldots, A_{k}\right)$ can be determined as a product

$$
\begin{aligned}
& F\left(A_{1}, A_{2}, \ldots, A_{k}\right)= {\left[A_{1}+A_{2}+\ldots+A_{k}\right] \cdot } \\
& \cdot\left[K \cdot A_{1}+K^{2} \cdot A_{2}+\ldots+K^{k} \cdot A_{k}\right] \cdot \\
& \vdots \\
& \\
& \cdot\left[K^{k-1} \cdot A_{1}+K^{k-2} \cdot A_{2}+\ldots+K \cdot A_{k}\right]
\end{aligned}
$$

Using only the symbols of the blocks i.e. $A_{1}, A_{2}, \ldots, A_{k}$ in (48), or in (49), we can analyze the formal function $F\left(A_{1}, A_{2}, \ldots, A_{k}\right)$. By replacing the symbols of the blocks with appropriate functions we get the solution

$$
g\left(s^{k}\right)=F\left[A_{1}(s), A_{2}(s), \ldots, A_{k}(s)\right] .
$$

The function (50) is called the 'block formula', and plays essential role in simplification of determination the function, and finally, the solution $g(s)$.

\section{Solution of the function $F$ for $k \leqslant 5$}

To simplicity the notation we slightly change the symbols of the blocks for $k \leqslant 5$ :

$$
A=A_{1}, B=A_{2}, C=A_{3}, D=A_{4}, E=A_{5} .
$$

Block formulas are designed for practical calculations of the problem discussed above. For any degree $n$, and given $k$, we can compute so called block function $F\left(A_{1}, A_{2}, \ldots, A_{k}\right)$. Below, there are presented four cases for $k=2,3,4,5$. The best readability of the formulas seems to be in a 'structure number notation' [2]. The columns show the product of the symbols. The square table shows the sum of columns (the product). 
1. $n \in N, k=1$

$$
F(A)=A
$$

2. $n \in N, k=2$

$$
F(A, B)=(A+B)(A-B)=A^{2}-B^{2}
$$

or, when expressing (53) in the 'structure number notation'

$$
F(A, B)=\left[\begin{array}{l}
A \\
A
\end{array}\right]-\left[\begin{array}{l}
B \\
B
\end{array}\right]
$$

3. $n \in N, k=3$

$$
F(A, B, C)=(A+B+C)\left(A^{2}+B^{2}+C^{2}-A B-A C-B C\right)=A^{3}+B^{3}+C^{3}-3 A B C
$$

or, when expressing (55) in the 'structure number notation'

$$
F(A, B, C)=\left[\begin{array}{lll}
A & B & C \\
A & B & C \\
A & B & C
\end{array}\right]-3 \cdot\left[\begin{array}{c}
A \\
B \\
C
\end{array}\right]
$$

4. $n \in N, k=4$

$$
F(A, B, C, D)=(A+B+C+D)(-A+B-C+D)\left(A^{2}+B^{2}+C^{2}+D^{2}-2 A C-2 B D\right)
$$

or when expanding and expressing (57) in the 'structure number notation'

$$
\begin{aligned}
& F(A, B, C, D)=\left[\begin{array}{cc}
B & D \\
B & D \\
B & D \\
B & D
\end{array}\right]-\left[\begin{array}{cc}
A & C \\
A & C \\
A & C \\
A & C
\end{array}\right]-4 \cdot\left[\begin{array}{cc}
A & A \\
B & D \\
B & D \\
C & C
\end{array}\right]+ \\
& 4 \cdot\left[\begin{array}{cc}
A & B \\
A & C \\
B & C \\
D & D
\end{array}\right]+2 \cdot\left[\begin{array}{c}
A \\
A \\
C \\
C
\end{array}\right]-2 \cdot\left[\begin{array}{c}
B \\
B \\
D \\
D
\end{array}\right]
\end{aligned}
$$

5. $n \in N, k=5$

$$
F(A, B, C, D, E)=X[Y-Z+T-R-3 a+2 b]
$$

where

$$
X=A+B+C+D+E
$$




$$
\begin{gathered}
Y=A^{4}+B^{4}+C^{4}+D^{4}+E^{4} \\
Z=A^{3}(B+C+D+E)+B^{3}(A+C+D+E)+ \\
C^{3}(A+B+D+E)+D^{3}(A+B+C+E)+E^{3}(A+B+C+D) \\
T=A^{2} B^{2}+A^{2} C^{2}+A^{2} D^{2}+A^{2} E^{2}+B^{2} C^{2}+B^{2} D^{2}+ \\
B^{2} E^{2}+C^{2} D^{2}+C^{2} E^{2}+D^{2} E^{2} \\
R=A B C D+A B C E+A B E F+A C D E+B C D E \\
a=A^{2}(D B+C E)+B^{2}(A C+D E)+C^{2}(A B+D E)+ \\
D^{2}(A B+C E)+E^{2}(A B+C D) \\
b=A^{2}(D+C)(B+E)+B^{2}(A+C)(D+E)+C^{2}(D+B)(A+E)+ \\
D^{2}(A+B)(C+E)+E^{2}(A+D)(B+C)
\end{gathered}
$$

or, when expanding and expressing (59) in the 'structure number notation'

$$
\begin{aligned}
F(A, B, C, D, E)= & {\left[\begin{array}{lllll}
A & B & C & D & E \\
A & B & C & D & E \\
A & B & C & D & E \\
A & B & C & D & E \\
A & B & C & D & E
\end{array}\right]-} \\
5 & {\left[\begin{array}{lllllllllll}
B & C & A & A & A & A & B & A & A & A & B \\
C & D & B & A & A & D & C & B & B & C & B \\
C & D & D & A & A & E & E & B & C & C & B \\
C & D & D & C & B & E & E & B & D & C & D \\
D & E & D & D & E & E & E & C & E & E & E
\end{array}\right]+} \\
5 \cdot & {\left[\begin{array}{llllllllll}
B & A & A & A & B & C & B & A & A & A \\
B & C & A & A & D & C & B & A & A & B \\
C & C & B & B & D & D & C & D & C & B \\
D & D & C & B & E & E & C & D & E & E \\
D & D & C & D & E & E & E & E & E & E
\end{array}\right] }
\end{aligned}
$$


In the above block formulas the polynomials $X, Y, Z, T, R$ are symmetric. The polynomials designated by $a$ and $b$ stand for non symmetric polynomials. They are so called 'cycling polynomials'. The block formulas for $k=1$ and $k=3$ are composed of symmetric polynomials only. Once we have the block formula for given $k$ we can compute $g(s)$ for given $f(s)$ regardless of its degree $n$. If $f(s)$ is composed of one block only, then $g(s)$ is reduced $f\left(s^{k}\right)$ ( $s^{k}$ should be replaced by $s$ ). There are other interesting corollaries resulting from the block formulas approach.

\section{Composition}

The relation $R_{k}$ can be composed as follows:

$$
R_{i} \circ R_{j}=R_{i j}
$$

The composition in this case is commutative and associative

$$
\begin{gathered}
R_{i} \circ R_{j}=R_{j} \circ R_{i} \\
R_{i} \circ\left(R_{j} \circ R_{l}\right)=\left(R_{j} \circ R_{i}\right) \circ R_{l}
\end{gathered}
$$

The above properties show that it is sufficient to determine the relation $R_{k}$ if $k$ is a prime number.

\section{Example}

For a given function $f(s)$ of the degree $n=7$ find the function $g(s)$ if $k=3$

$$
f(s)=a_{0} \cdot s^{7}+a_{1} s^{6}+a_{2} s^{5}+a_{3} s^{4}+a_{4} s^{3}+a_{5} s^{2}+a_{6} s+a_{7}, \quad a_{0}=1 .
$$

When applying any of the four described methods we find the monomials in coefficients of $g(s)$ with appropriate numerical (integer) coefficients. They can be arranged and classified. A proper way to graphically present such an arrangement is to design it around the indexes of $a_{i}$, like in a structure number.

$$
\begin{gathered}
-3 \cdot\left[\begin{array}{cccccccccccccccccc}
0 & 0 & 0 & 0 & 0 & 0 & 3 & 3 & 3 & 3 & 3 & 3 & 6 & 6 & 6 & 6 & 6 & 6 \\
1 & 1 & 4 & 4 & 7 & 7 & 1 & 1 & 4 & 4 & 7 & 7 & 1 & 1 & 4 & 4 & 7 & 7 \\
2 & 5 & 2 & 5 & 2 & 5 & 2 & 5 & 2 & 5 & 2 & 5 & 2 & 5 & 2 & 5 & 2 & 5
\end{array}\right], \\
1 \cdot\left[\begin{array}{cccccccc}
0 & 1 & 2 & 3 & 4 & 5 & 6 & 7 \\
0 & 1 & 2 & 3 & 4 & 5 & 6 & 7 \\
0 & 1 & 2 & 3 & 4 & 5 & 6 & 7
\end{array}\right],
\end{gathered}
$$




$$
\begin{gathered}
3 \cdot\left[\begin{array}{llllllllllllll}
0 & 0 & 3 & 3 & 6 & 6 & 1 & 1 & 4 & 4 & 7 & 7 & 2 & 5 \\
0 & 0 & 3 & 3 & 6 & 6 & 1 & 1 & 4 & 4 & 7 & 7 & 2 & 5 \\
3 & 6 & 0 & 6 & 0 & 3 & 7 & 4 & 1 & 7 & 4 & 1 & 5 & 2
\end{array}\right] \\
6 \cdot\left[\begin{array}{ll}
0 & 1 \\
3 & 4 \\
6 & 7
\end{array}\right]
\end{gathered}
$$

The above 42 monomials of $b_{i}$, written in the form of indexes in columns, can be classified in many different ways. They show significant symmetry. Symbol $\Rightarrow$ presents appropriate mapping. If one takes the numerical coefficients (72), (73), (74) and (75) with appropriate sign they should be understood in the way as e.g for (77): $b_{1}=$ $-3 \cdot a_{0} a_{1} a_{2}+a_{1}^{3}+3 \cdot a_{0}^{2} a_{3}$.

$$
\begin{aligned}
& b_{0} \Rightarrow\left[\begin{array}{l}
0 \\
0 \\
0
\end{array}\right]\left[\begin{array}{l}
7 \\
7 \\
7
\end{array}\right] \Leftarrow b_{7} \\
& b_{1} \Rightarrow\left[\begin{array}{lll}
0 & 1 & 0 \\
1 & 1 & 0 \\
2 & 1 & 3
\end{array}\right]\left[\begin{array}{lll}
7 & 6 & 7 \\
6 & 6 & 7 \\
5 & 6 & 4
\end{array}\right] \Leftarrow b_{6} \\
& b_{2} \Rightarrow\left[\begin{array}{lllllll}
0 & 0 & 3 & 2 & 0 & 3 & 1 \\
1 & 4 & 1 & 2 & 0 & 3 & 1 \\
5 & 2 & 2 & 2 & 6 & 0 & 4
\end{array}\right] \quad\left[\begin{array}{lllllll}
7 & 7 & 4 & 5 & 7 & 4 & 6 \\
6 & 3 & 6 & 5 & 7 & 4 & 6 \\
2 & 5 & 5 & 5 & 1 & 7 & 3
\end{array}\right] \Leftarrow b_{5} \\
& b_{3} \Rightarrow\left[\begin{array}{llllllllll}
0 & 0 & 3 & 3 & 6 & 3 & 1 & 4 & 2 & 0 \\
4 & 7 & 1 & 4 & 1 & 3 & 1 & 4 & 2 & 3 \\
5 & 2 & 5 & 2 & 2 & 3 & 7 & 1 & 5 & 6
\end{array}\right] \quad\left[\begin{array}{llllllllll}
7 & 7 & 4 & 5 & 1 & 4 & 6 & 3 & 4 & 7 \\
3 & 0 & 6 & 3 & 6 & 4 & 6 & 3 & 4 & 4 \\
2 & 5 & 2 & 5 & 5 & 4 & 0 & 6 & 2 & 1
\end{array}\right] \Leftarrow b_{4}
\end{aligned}
$$

In the structure number notation the symmetry in indexes becomes much more visible. In the above example the following property is true:

$$
b_{i}\left(a_{0}, a_{1}, a_{2}, a_{3}, a_{4}, a_{5}, a_{6}, a_{7}\right)=b_{7-i}\left(a_{7}, a_{6}, a_{5}, a_{4}, a_{3}, a_{2}, a_{1}, a_{0}\right)
$$

Also the numerical coefficients are identical - see Tab. 2. This symmetry can be generalized for any $n$, and any $k$. Thus, the solution is

$$
b_{i}\left(a_{0}, a_{1}, a_{2}, a_{3}, \ldots, a_{n-1}, a_{n}\right)=b_{n-i}\left(a_{n}, a_{n-1}, \ldots, a_{3}, a_{2}, a_{1}, a_{0}\right), \quad n \in N, \quad i=1,2, \ldots, n .
$$


Table 5. Symmetry of the coefficients.

\begin{tabular}{|l|l|l|r|}
\hline \hline & & $n=7 \quad k=3$ & \\
\hline \hline$b_{0}$ & $=$ & $\left(a_{0}\right)^{3}$ & $(82)$ \\
\hline$b_{1}$ & $=$ & $-3 a_{0} a_{1} a_{2}+\left(a_{1}\right)^{3}+3\left(a_{0}\right)^{2} a_{3}$ & $(83)$ \\
\hline$b_{2}$ & $=-3 a_{0} a_{1} a_{5}-3 a_{0} a_{4} a_{2}-3 a_{3} a_{1} a_{2}+\left(a_{2}\right)^{3}+3\left(a_{0}\right)^{2} a_{6}+3\left(a_{3}\right)^{2} a_{0}+3\left(a_{1}\right)^{2} a_{4}$ & $(84)$ \\
\hline$b_{3}$ & $=\begin{array}{l}-3 a_{0} a_{4} a_{5}-3 a_{0} a_{7} a_{2}-3 a_{3} a_{1} a_{5}-3 a_{3} a_{4} a_{2}-3 a_{6} a_{1} a_{2}+\left(a_{3}\right)^{3}+3\left(a_{1}\right)^{2} a_{7}+ \\
3\left(a_{4}\right)^{2} a_{1}+3\left(a_{2}\right)^{2} a_{5}+6 a_{0} a_{3} a_{6}\end{array}$ & $(85)$ \\
\hline$b_{4}$ & $=\begin{array}{l}-3 a_{7} a_{3} a_{2}-3 a_{7} a_{0} a_{5}-3 a_{4} a_{6} a_{2}-3 a_{4} a_{3} a_{5}-3 a_{1} a_{6} a_{5}+\left(a_{4}\right)^{3}+3\left(a_{6}\right)^{2} a_{0}+ \\
3\left(a_{3}\right)^{2} a_{6}+3\left(a_{5}\right)^{2} a_{2}+6 a_{7} a_{4} a_{1}\end{array}$ & $(86)$ \\
\hline$b_{5}$ & $=-3 a_{7} a_{6} a_{2}-3 a_{7} a_{3} a_{5}-3 a_{4} a_{6} a_{2}+\left(a_{5}\right)^{3}+3\left(a_{7}\right)^{2} a_{1}+3\left(a_{4}\right)^{2} a_{7}+3\left(a_{6}\right)^{2} a_{3}$ & $(87)$ \\
\hline$b_{6}$ & $=-3 a_{7} a_{6} a_{5}+\left(a_{6}\right)^{3}+3\left(a_{7}\right)^{2} a_{4}$ & $(88)$ \\
\hline$b_{7}$ & $=\left(a_{7}\right)^{3}$ & \\
\hline \hline
\end{tabular}

\section{Other relations}

We can apply symmetric polynomials [11] to solve a large set of other problems. For example, given the polynomial

$$
s^{3}+a_{1} \cdot s^{2}+a_{2} \cdot s+a_{3}
$$

with the roots

$$
U=\left\{s_{1}, s_{2}, s_{3}\right\}
$$

find a polynomial

$$
s^{3}+c_{1} \cdot s^{2}+c_{2} \cdot s+c_{3}
$$

where the roots are

$$
W=\left\{s_{1}+s_{2}, s_{1}+s_{3}, s_{2}+s_{3}\right\} .
$$

Solution:

The coefficients of (90) in a spectral notation take the following form:

$$
a_{1}=-((1)), \quad a_{2}=-((1,1)), \quad a_{3}=-((1,1,1)) .
$$

Polynomial (92) can be written as

$$
\left(s-s_{1}-s_{2}\right) \cdot\left(s-s_{1}-s_{3}\right) \cdot\left(s-s_{2}-s_{3}\right)
$$

When expanding (95) we get the coefficients $c_{1}, c_{2}, c_{3}$

$$
c_{1}=-2 \cdot\left(s_{1}+s_{2}+s_{3}\right)
$$




$$
\begin{gathered}
c_{2}=s_{1}^{2}+s_{2}^{2}+s_{3}^{2}+3 \cdot\left(s_{1} \cdot s_{2}+s_{1} \cdot s_{3}+s_{2} \cdot s_{3}\right) \\
c_{3}=-s_{1}^{2} \cdot s_{2}-s_{1}^{2} \cdot s_{3}-s_{2}^{2} \cdot s_{1}-s_{2}^{2} \cdot s_{3}-s_{3}^{2} \cdot s_{1}-s_{3}^{2} \cdot s_{2}-2 \cdot s_{1} \cdot s_{2} \cdot s_{3}
\end{gathered}
$$

In the spectral notation the coefficients (96) - (98) can be written as

$$
c_{1}=-2 \cdot((1)), \quad c_{2}=((2))+3 \cdot((1,1)), \quad c_{3}=-((2,1))-2 \cdot((1,1,1)) .
$$

In the theory presented in [1] we have the identities

$$
((2))=-2 \cdot((1,1))+((1))^{2}, \quad((2,1))=-3 \cdot((1,1,1))+((1,1)) \cdot((1)) .
$$

Substituting (100) to (99) one gets

$$
c_{1}=-2 \cdot((1)), \quad c_{2}=((1))^{2}+((1,1)), \quad c_{3}=((1,1,1))-((1,1)) \cdot((1)) .
$$

When replacing spectral symbols in (99) by standard symbols of coefficients we get

$$
c_{1}=2 \cdot a_{1}, \quad c_{2}=a_{1}^{2}+a_{2}, \quad c_{3}=-a_{3}+a_{2} \cdot a_{1} .
$$

Thus the following polynomial satisfies (92)

$$
c_{1}=-2 \cdot((1)), \quad c_{2}=((1))^{2}+((1,1)), \quad c_{3}=((1,1,1))-((1,1)) \cdot((1)) .
$$

\section{Comments}

Four methods of determining the relation $R_{k}$, and their improvement (block decomposition) can be applied for any $n$, and theoretically for any $k$. Analysis allows to formulate a set of theoretical properties and rules.

1. Coefficients $b_{i}$ are composed of appropriate monomials with numerical, integer coefficients.

2. Integer coefficients can be determined by analyzing the block decomposition. They are connected with a generalized Pascal triangle and are associated with $\left(\left(r_{1}, r_{2}, \ldots, r_{i}\right)\right)$.

3. It is sufficient to know the solutions for $k$ only, where $k$ is a prime number. Once we know the integer coefficients in the block function $F\left(A_{1}, A_{2}, \ldots, A_{k}\right)$ which are always integer, we are able to determine the coefficients of all monomials.

4. The above properties constitute a base to elaborate theoretical procedure (the fifth method) which delivers numerical coefficients.

5. The problem of finding the relation $R_{k}$ is only an example of a much larger set of problems. One of such problem is presented in sec. 11.

6. Full set of integer coefficients appears if the degree of $f(s)$ equals $k^{2}$. 
Table 6. Solutions for chosen $n \leqslant 5$ and $k \leqslant 5$.

\begin{tabular}{|l|l|}
\hline \hline \multicolumn{1}{|c|}{$k=2$} & \multicolumn{1}{c|}{$k=3$} \\
\hline \hline$b_{1}=2 a_{2}-\left(a_{1}\right)^{2}$ & $b_{1}=-3 a_{1} a_{2}+\left(a_{1}\right)^{3}$ \\
\hline$b_{2}=\left(a_{2}\right)^{2}$ & $b_{2}=\left(a_{2}\right)^{3}$ \\
\hline \hline$b_{1}=-\left(a_{1}\right)^{2}+2 a_{2}$ & $b_{1}=-3 a_{1} a_{2}+\left(a_{1}\right)^{3}+3 a_{3}$ \\
\hline$b_{2}=\left(a_{2}\right)^{2}-2 a_{1} a_{3}$ & $b_{2}=3\left(a_{3}\right)^{2}-3 a_{1} a_{2} a_{3}+\left(a_{2}\right)^{3}$ \\
\hline$b_{3}=-\left(a_{3}\right)^{2}$ & $b_{3}=\left(a_{3}\right)^{3}$ \\
\hline \hline$b_{1}=-\left(a_{1}\right)^{2}+2 a_{2}$ & $b_{1}=-3 a_{1} a_{2}+\left(a_{1}\right)^{3}+3 a_{3}$ \\
\hline$b_{2}=2 a_{4}+\left(a_{2}\right)^{2}-2 a_{1} a_{3}$ & $b_{2}=3\left(a_{1}\right) 2 a_{4}+3\left(a_{3}\right) 2-3 a_{1} a_{2} a_{3}-3 a_{2} a_{4}+\left(a_{2}\right)^{3}$ \\
\hline$b_{3}=-\left(a_{3}\right)^{2}+2 a_{4} a_{2}$ & $b_{3}=\left(a_{3}\right)^{3}-3 a_{2} a_{3} a_{4}+3 a_{1}\left(a_{4}\right)^{2}$ \\
\hline$b_{4}=\left(a_{4}\right)^{2}$ & $b_{4}=\left(a_{4}\right)^{3}$ \\
\hline \hline$b_{1}=-\left(a_{1}\right)^{2}+2 a_{2}$ & $b_{1}=-3 a_{1} a_{2}+\left(a_{1}\right)^{3}+3 a_{3}$ \\
\hline$b_{2}=2 a_{4}+\left(a_{2}\right)^{2}-2 a_{1} a_{3}$ & $b_{2}=-3 a_{1} a_{5}-3 a_{2} a_{4}+3\left(a_{1}\right) 2 a_{4}-3 a_{1} a_{2} a_{3}+$ \\
\hline$b_{3}=-\left(a_{3}\right)^{2}-2 a_{1} a_{5}+2 a_{4} a_{2}$ & $b_{3}=-3 a_{1} a_{3} a_{5}+\left(a_{3}\right)^{3}-3 a_{2} a_{3} a_{4}+3 a_{1}\left(a_{4}\right)^{2}+$ \\
\hline$b_{4}=\left(a_{4}\right)^{2}-2 a_{5} a_{3}$ & $b_{4}=-3 a_{3} a_{4} a_{5}+\left(a_{4}\right)^{3}+3 a_{2}\left(a_{5}\right)^{2}$ \\
\hline$b_{5}=-\left(a_{5}\right)^{2}$ & $b_{5}=\left(a_{5}\right)^{3}$ \\
\hline \hline
\end{tabular}

\section{Appendix}

The spectral notation used in the Theory of Symmetric Polynomials allows to find unlimited number of identities. The simplest two examples of this kind are the following:

$$
\begin{gathered}
((1)) 2=((2))+2((1,1)), \\
((1)) 3=((3))+3((2,1))+6((1,1,1)) .
\end{gathered}
$$

In the standard notation both of the above expressions are understood as

$$
\begin{gathered}
\left(x_{1}+x_{2}+\ldots\right)^{2}=\left(x_{1}\right)^{2}+\left(x_{2}\right)^{2}+\cdots+2\left(x_{1} x_{2}+x_{1} x_{3}+\ldots\right), \\
\left(x_{1}+x_{2}+\ldots\right)^{3}=\left(x_{1}\right)^{3}+\left(x_{2}\right)^{3}+\cdots+3\left[\left(x_{1}\right) 2_{2}^{x}+\left(x_{1}\right)^{2} x_{3}+\ldots\right]+6\left(x_{1} x_{2} x_{3}+x_{1} x_{2} x_{4}+\ldots\right) .
\end{gathered}
$$

The spectral theory delivers effective procedures which allow to find symmetric polynomials as functions:

$$
f[((1)),((1,1)),((1,1,1)), \ldots],
$$


Table 3. Cont.

\begin{tabular}{|c|}
\hline$k=4$ \\
\hline$b_{1}=-2\left(a_{2}\right)^{2}+4 a_{2}\left(a_{1}\right)^{2}-\left(a_{1}\right)^{4}$ \\
\hline$b_{2}=\left(a_{2}\right)^{4}$ \\
\hline$b_{1}=-4 a_{1} a_{3}+4 a_{2}\left(a_{1}\right)^{2}-(a 1)^{4}-2\left(a_{2}\right)^{2}$ \\
\hline$b_{2}=2\left(a_{1}\right)^{2}\left(a_{3}\right)^{2}+\left(a_{2}\right)^{4}+4\left(a_{3}\right)^{2} a_{2}-4 a_{1} a_{3}\left(a_{2}\right)^{2}$ \\
\hline$b_{3}=-\left(a_{3}\right)^{4}$ \\
\hline$b_{1}=-4 a_{1} a_{3}-2\left(a_{2}\right)^{2}+4 a_{2}\left(a_{1}\right)^{2}-\left(a_{1}\right)^{4}+4 a_{4}$ \\
\hline $\begin{aligned} b_{2}=-8 a_{1} a_{3} a_{4}-4 a_{1} a_{3}\left(a_{2}\right)^{2}-4 a_{4}\left(a_{2}\right)^{2}+4 a_{4} a_{2}\left(a_{1}\right)^{2}+2\left(a_{1}\right)^{2}\left(a_{3}\right)^{2}+4\left(a_{3}\right)^{2} a_{2}+ \\
\quad 6\left(a_{4}\right)^{2}+\left(a_{2}\right)^{4}\end{aligned}$ \\
\hline$b_{3}=4\left(a_{4}\right)^{3}+4 a_{4}\left(a_{3}\right)^{2} a_{2}-\left(a_{3}\right)^{4}-4\left(a_{4}\right)^{2} a_{1} a_{3}-2\left(a_{4}\right)^{2}\left(a_{2}\right)^{2}$ \\
\hline$b_{4}=\left(a_{4}\right)^{4}$ \\
\hline$b_{1}=-4 a_{1} a_{3}-2\left(a_{2}\right)^{2}+4 a_{2}\left(a_{1}\right)^{2}-\left(a_{1}\right)^{4}+4 a_{4}$ \\
\hline $\begin{aligned} b_{2}= & 8 a_{1} a_{2} a_{5}+\left(a_{2}\right)^{4}-4 a_{4}\left(a_{2}\right)^{2}-4 a_{1} a_{3}\left(a_{2}\right)^{2}+4\left(a_{3}\right)^{2} a_{2}-8 a_{4} a_{1} a_{3}-4\left(a_{1}\right)^{3} a_{5}+ \\
& 6\left(a_{4}\right)^{2}-4 a_{3} a_{5}+4 a_{4} a_{2}\left(a_{1}\right)^{2}+2\left(a_{1}\right)^{2}\left(a_{3}\right)^{2}\end{aligned}$ \\
\hline $\begin{aligned} b_{3}= & a_{4} a_{1} a_{2} a_{5}-4\left(a_{4}\right)^{2} a_{1} a_{3}+4 a_{4}\left(a_{3}\right)^{2} a_{2}-4 a_{5} a_{3}\left(a_{2}\right)^{2}+4 a_{1}\left(a_{3}\right)^{2} a_{5}+4\left(a_{4}\right)^{3}- \\
& 6\left(a_{1}\right)^{2}\left(a_{5}\right)^{2}+4 a_{2}\left(a_{5}\right)^{2}-\left(a_{3}\right)^{4}-2\left(a_{4}\right)^{2}\left(a_{2}\right)^{2}-8 a_{4} a_{3} a_{5}\end{aligned}$ \\
\hline$b_{4}=-4\left(a_{4}\right)^{2} a_{3} a_{5}+\left(a_{4}\right)^{4}+4 a_{4} a_{2}\left(a_{5}\right)^{2}+2\left(a_{3}\right)^{2}\left(a_{5}\right)^{2}-4 a_{1}\left(a_{5}\right)^{3}$ \\
\hline$b_{5}=-\left(a_{5}\right)^{4}$ \\
\hline
\end{tabular}

$$
g[((1)),((2)),((3)) \ldots] .
$$

For example

$$
\begin{gathered}
((2,1))=-3((1,1,1))+((1,1))((1)) \\
((2,1))=-((3))+((2))((1)) \\
((2))((1))=-2((1,1))((1))+((1))^{3} \\
((1,1))((1))=-1 / 2((2))((1))+1 / 2((1))^{3}
\end{gathered}
$$

The above examples of identities are computed as results of the transformations in a vector space. The basic transformations for $n=2$ and $n=3$ are presented below:

$$
\left[\begin{array}{c}
((1,1)) \\
((1))^{2}
\end{array}\right]=\left[\begin{array}{ll}
1 & 0 \\
2 & 1
\end{array}\right] \cdot\left[\begin{array}{c}
((1,1)) \\
((2))
\end{array}\right],
$$


Table 3. Cont.

\begin{tabular}{|c|}
\hline$k=5$ \\
\hline$b_{1}=-5 a_{2}\left(a_{1}\right)^{3}+\left(a_{1}\right)^{5}+5 a_{1}\left(a_{2}\right)^{2}$ \\
\hline$b_{2}=\left(a_{2}\right)^{5}$ \\
\hline$b_{1}=5 a_{3}\left(a_{1}\right)^{2}+5 a_{1}\left(a_{2}\right)^{2}-5 a_{2}\left(a_{1}\right)^{3}-5 a_{3} a_{2}+\left(a_{1}\right)^{5}$ \\
\hline$b_{2}=-5 a_{1}\left(a_{3}\right)^{3}-5 a_{3}\left(a_{2}\right)^{3} a_{1}+5\left(a_{3}\right)^{2} a_{2}\left(a_{1}\right)^{2}+\left(a_{2}\right)^{5}+5\left(a_{3}\right)^{2}\left(a_{2}\right)^{2}$ \\
\hline$b_{3}=\left(a_{3}\right)^{5}$ \\
\hline $\begin{aligned} b_{1}= & \left(a_{1}\right)^{5}-5 a_{3} a_{2}-5 a_{2}\left(a_{1}\right)^{3}+5 a_{3}\left(a_{1}\right)^{2}-5 a_{1} a_{4}+5 a_{1}\left(a_{2}\right)^{2}+5\left(a_{3}\right)^{2}\left(a_{2}\right)^{2}- \\
& 5 a_{1}\left(a_{3}\right)^{3}+5\left(a_{1}\right)^{2}\left(a_{4}\right)^{2}\end{aligned}$ \\
\hline $\begin{aligned} b_{2}= & -5 a_{4}\left(a_{2}\right)^{3}+5\left(a_{3}\right)^{2} a_{2}\left(a_{1}\right)^{2}+\left(a_{2}\right)^{5}+5\left(a_{1}\right)^{2} a_{4}\left(a_{2}\right)^{2}-5 a_{1} a_{4} a_{3} a_{2}+5\left(a_{4}\right)^{2} a_{2}+ \\
& 5 a_{4}\left(a_{3}\right)^{2}-5\left(a_{1}\right)^{3} a_{4} a_{3}-5 a_{3}\left(a_{2}\right)^{3} a_{1}\end{aligned}$ \\
\hline$b_{3}=-5 a_{1}\left(a_{4}\right)^{3} a_{2}-5\left(a_{3}\right)^{3} a_{2} a_{4}+5 a_{1}\left(a_{4}\right)^{2}\left(a_{3}\right)^{2}+5 a_{3}\left(a_{2}\right)^{2}\left(a_{4}\right)^{2}+\left(a_{3}\right)^{5}-5\left(a_{4}\right)^{3} a_{3}$ \\
\hline$b_{4}=\left(a_{4}\right)^{5}$ \\
\hline$b_{1}=-5 a_{1} a_{4}+5 a_{1}\left(a_{2}\right)^{2}-5 a_{2}\left(a_{1}\right)^{3}-5 a_{3} a_{2}+5 a_{3}\left(a_{1}\right)^{2}+\left(a_{1}\right)^{5}+5 a_{5}$ \\
\hline $\begin{aligned} b_{2}= & -5 a_{5} a_{2}\left(a_{1}\right)^{3}-15 a_{5} a_{1} a_{4}+10 a_{5} a_{3}\left(a_{1}\right)^{2}-15 a_{5} a_{3} a_{2}+5\left(a_{3}\right)^{2}\left(a_{2}\right)^{2}+5\left(a_{4}\right)^{2} a_{2}+ \\
& 5 a_{4}\left(a_{3}\right)^{2}-5 a_{4}\left(a_{2}\right)^{3}+5\left(a_{1}\right)^{2}\left(a_{4}\right)^{2}-a_{1}\left(a_{3}\right)^{3}+10\left(a_{5}\right)^{2}+\left(a_{2}\right)^{5}-5\left(a_{1}\right)^{3} a_{4} a_{3}- \\
& 5 a_{1} a_{4} a_{2} a_{3}+5\left(a_{1}\right)^{2} a_{4}\left(a_{2}\right)^{2}-5 a_{3}\left(a_{2}\right)^{2} a_{1}+5\left(a_{3}\right)^{2} a_{2}\left(a_{1}\right)^{2}+10 a_{5} a_{1}\left(a_{2}\right)^{2}\end{aligned}$ \\
\hline $\begin{aligned} b_{3}= & -5\left(a_{4}\right)^{3} a_{3}+10 a_{5} a_{4}\left(a_{3}\right)^{2}-5 a_{5} a_{1} a_{4} a_{3} a_{2}+5 a_{5}\left(a_{1}\right)^{2}\left(a_{4}\right)^{2}+5 a_{3}\left(a 2^{2}\left(a_{4}\right)^{2}-\right. \\
& 5\left(a_{3}\right)^{3} a_{2} a_{4}-5 a_{5} a_{1}\left(a_{3}\right)^{3}+5 a_{5}\left(a_{3}\right)^{2}\left(a_{2}\right)^{2}-5 a_{5} a_{4}\left(a_{2}\right)^{3}+10 a_{5}\left(a_{4}\right)^{2} a_{2}+ \\
& 5 a_{1}\left(a_{4}\right)^{2}\left(a_{3}\right)^{2}-5 a_{1}\left(a_{4}\right)^{3} a_{2}+5\left(a_{5}\right)^{2} a_{1}\left(a_{2}\right)^{2}-15\left(a_{5}\right)^{2} a_{3} a_{2}+5\left(a_{5}\right)^{2} a_{3}\left(a_{1}\right)^{2}- \\
& 15\left(a_{5}\right)^{2} a_{1} a_{4}+10\left(a_{5}\right)^{3}+\left(a_{3}\right)^{5}\end{aligned}$ \\
\hline $\begin{aligned} b_{4}= & -5 a_{5}\left(a_{4}\right)^{3} a_{3}-5\left(a_{5}\right)^{3} a_{3} a_{2}-5\left(a_{5}\right)^{3} a_{1} a_{4}+5\left(a_{5}\right)^{2} a_{4}\left(a_{3}\right)^{2}+5\left(a_{5}\right)^{2}\left(a_{4}\right)^{2} a_{2}+ \\
& 5\left(a_{5}\right)^{4}+\left(a_{4}\right)^{5}\end{aligned}$ \\
\hline$b_{5}=\left(a_{5}\right)^{5}$ \\
\hline
\end{tabular}

$$
\begin{gathered}
{\left[\begin{array}{c}
((2)) \\
((1))^{2}
\end{array}\right]=\left[\begin{array}{ll}
0 & 1 \\
1 & 2
\end{array}\right] \cdot\left[\begin{array}{c}
((1,1)) \\
((2))
\end{array}\right],} \\
{\left[\begin{array}{c}
((1,1,1)) \\
((1,1))((1)) \\
((1))^{3}
\end{array}\right]=\left[\begin{array}{lll}
1 & 0 & 0 \\
3 & 1 & 0 \\
6 & 3 & 1
\end{array}\right] \cdot\left[\begin{array}{c}
((1,1,1)) \\
((2,1)) \\
((3))
\end{array}\right],}
\end{gathered}
$$




$$
\left[\begin{array}{c}
((3)) \\
((2))((1)) \\
((1))^{3}
\end{array}\right]=\left[\begin{array}{lll}
0 & 0 & 1 \\
0 & 1 & 1 \\
6 & 3 & 1
\end{array}\right] \cdot\left[\begin{array}{c}
((1,1,1)) \\
((2,1)) \\
((3))
\end{array}\right]
$$

When calculating the inverse transformations we get a set of new formulas. When we perform the compositions of the appropriate transformations we arrive at the complete set of formulas. The matrix formulas (20), (21) and (23) which can be found in [6], are essential to calculate particular set of spectral formulas. For the power $n=4$ the dimension of the vector space $d(4)=5$. For the power $n=50$ the dimension of the vector space $d(50)=204226$.

\section{References}

[1] J. Ambrosiewicz: About certain generalization of associated matrix. ZN WSP, Katowice, 1967, (in Polish).

[2] S. BELERT and H. WoŹNIACKI: Analisis and synthesis of electrical systems using structural numbers method. WNT, Warsaw, 1968, (in Polish).

[3] D. Cox, J. Little and D. O'SheA: Ideals, varieties and algorithms, An introduction to computational algebraic geometry and commutative algebra. Springer New York, 1998.

[4] A. Cayley: The Collected Mathematical Papers. XII Cambridge, 1897.

[5] J. FlachSmeYer: Kombinatorik, Eine Einfuerung in die mengenteoretische Denkweise. VEB Deutscher Verlag der Wissenschaften, Berlin, 1969.

[6] A. Kourosh: Higher algebra. Mir Publisher, Moscow, 1972.

[7] B.Noble, J.W.Daniel: Applied Linear Algebra. Prentice-Hall, Englewood Cliffs, New Jersey. 1977

[8] P. Nowacki, L. SzKlarski and H. GóRecki: Principles of control system theory. PWN, Warszawa, 1958, (in Polish).

[9] Z. OPIAL: Higher algebra. PWN, Warszawa, 1969, (in Polish).

[10] T. PiwowarczyK: Simplification of rational functions. Cracow University of Technology, Z 17 Cracow, (2008).

[11] T. PIWOWARCZYK: Multipower notation of symmetric polynomials in engineering calculus. PAN, Kraków, 2000.

[12] T. PiwowARCZYK: The theory of finite numerical sequences in the analysis of electrical circuits. CCNS, Cracow, 2002, (in Polish). 
[13] T. PiwowarczyK: Symmetric polynomials of several variables in electric circuits. Cracow University of Thechnology, Cracow, 2002, (in Polish).

[14] A. Turowicz: The geometry of zeros of polynomials. PWN, Warsaw, 1967, (in Polish). 